\title{
QUASI $s$-NUMBERS AND MEASURES OF NON-COMPACTNESS OF MULTILINEAR OPERATORS
}

\author{
Dicesar L. Fernandez, Mieczysław Mastyło and Eduardo B. da Silva \\ Universidade Estadual de Campinas - Unicamp, Instituto de Matemática \\ Campinas, São Paulo, 13083-859, Brazil; dicesar@ime.unicamp.br \\ Adam Mickiewicz University, Faculty of Mathematics \& Computer Science \\ and Polish Academy of Science (Poznań branch), Institute of Mathematics \\ Umultowska 87, 61-614 Poznań, Poland; mastylo@amu.edu.pl \\ Universidade Estadual de Maringá - UEM, Departamento de Matemática \\ Av. Colombo 5790, Maringá, Paraná, 870300-110, Brazil; ebsilva@wnet.com.br
}

\begin{abstract}
The main goal of this paper is the study of quasi $s$-numbers of multilinear operators among Banach spaces. The relationships among multilinear variants of approximation, Kolmogorov and Gelfand numbers of operators and their generalized linear adjoint are shown. In the multilinear case, analogous theorems which are well-known in the linear case, are stated and proved. The estimates of measures of non-compactness of multilinear operators in terms of measures of the adjoint operators are also proved.
\end{abstract}

\section{Introduction}

The theory of $s$-numbers of linear bounded operators among Banach spaces was introduced and studied by Pietsch [10]. It plays a fundamental role in the theory of operators and the local theory of Banach spaces and it is a powerful tool in the study of eigenvalue distribution of Riesz operators in Banach spaces (see, e.g., [9, 13]). In 1983 Pietsch [12] proposed and sketched a theory of ideals and $s$-numbers of multilinear functionals. While the properties of $s$-numbers of linear operators have been studied extensively, the theory of $s$-numbers of multilinear operators has not been studied yet.

In this paper the theory of quasi s-number sequences of bounded multilinear operators among Banach spaces is developed. We investigate the question of how the fundamental properties of important $s$-numbers of linear operators are inherited to the multilinear case. It should be noted that whereas the work is based on some ideas from the theory of $s$-numbers of bounded linear operators, some proofs may be extended from the linear case to the multilinear operators and other require new ideas and methods. The difficulty comes from the fact that even in the bilinear case the range or the kernel of a bilinear operator is not necessarily a linear subspace. In

doi:10.5186/aasfm.2013.3842

2010 Mathematics Subject Classification: Primary 46G25, 47A07, 47B06.

Key words: Multilinear operator, s-numbers, approximation, Gelfand, Kolmogorov numbers, the measure of non-compactness.

The first author was partially supported by FAPESP grant Proc. 2011/51602-5, the second author was partially supported by the National Science Centre (NCN), Poland, grant no. 2011/01/B/ST1/06243 and the third author was partially supported by FAPESP grant Proc. $2011 / 51602-7$. 
particular, as a consequence the well-known relations between the dimensions of the kernel and the range in the linear case are not true in general in the multilinear case.

Throughout the paper the standard notation from the Banach space theory is used. If $X$ is a Banach space we denote by $X^{*}$ its dual Banach space and by $U_{X}, \stackrel{\circ}{U}_{X}$ the closed and open unit balls of $X$, respectively. As usual $\kappa_{X}$ denotes the canonical embedding of $X$ to the bidual $X^{* *}$ of $X$. For each $m \in \mathbf{N}$ the product $X_{1} \times \cdots \times X_{m}$ of Banach spaces is equipped with the norm $\left\|\left(x_{1}, \ldots, x_{m}\right)\right\|=\max _{1 \leq j \leq m}\left\|x_{j}\right\|_{X_{j}}$. We shall denote by $\mathcal{L}_{m}\left(X_{1} \times \cdots \times X_{m}, Y\right)$ the Banach space of all $m$-linear bounded operators defined on $X_{1} \times \cdots \times X_{m}$ with values in a Banach space $Y$, equipped with the norm

$$
\|T\|=\sup \left\{\left\|T\left(x_{1}, \ldots, x_{m}\right)\right\|_{Y} ;\left(x_{1}, \ldots, x_{m}\right) \in U_{X_{1}} \times \cdots \times U_{X_{m}}\right\} .
$$

In the case when $m=1$, we shall write $\mathcal{L}\left(X_{1}, Y\right)$ instead of $\mathcal{L}_{1}\left(X_{1}, Y\right)$. In the case when $Y$ is the scalar field $\mathbf{K}(\mathbf{K}=\mathbf{R}$ or $\mathbf{K}=\mathbf{C})$, we denote the space of all $m$-linear forms by $\mathcal{L}_{m}\left(X_{1} \times \cdots \times X_{m}\right)$. As usual, $X_{1} \widehat{\otimes}_{\pi} \cdots \widehat{\otimes}_{\pi} X_{m}$ will denote the projective tensor product of the Banach spaces $X_{1}, \ldots, X_{m}$.

For $m \geq 2$ let $X_{1}, \ldots, X_{m}$ be Banach spaces. Following [6] the Banach space $X_{1} \times \cdots \times X_{m}$ is said to have the multilinear extension property if, whenever $F_{i} \subset X_{i}$ $(1 \leq i \leq m)$ are closed subspaces and $T \in \mathcal{L}_{m}\left(F_{1} \times \cdots \times F_{m}\right)$ is a continuous multilinear form, then there exists a continuous multilinear form $\widetilde{T} \in \mathcal{L}_{m}\left(X_{1} \times \cdots \times X_{m}\right)$ such that $\left.\widetilde{T}\right|_{F_{1} \times \cdots \times F_{m}}=T$. When $m=2$, we will also say that $X_{1} \times X_{2}$ has the bilinear extension property. It was proved in [6, Theorem 2.3] that $X_{1} \times \cdots \times X_{m}$ has the multilinear extension property if, and only if, there exists a constant $M>0$ such that whenever $F_{i} \subset X_{i}$ are closed subspaces, every $\widetilde{T} \in \mathcal{L}_{m}\left(F_{1} \times \cdots \times F_{m}\right)$ has an extension $\widetilde{T} \in \mathcal{L}_{m}\left(X_{1} \times \cdots \times X_{m}\right)$ with $\|\widetilde{T}\| \leq M\|T\|$. In this case we say that the $X_{1} \times \cdots \times X_{m}$ has the multilinear extension property with constant $M$, and the least constant is denoted by $M\left(X_{1} \times \cdots \times X_{m}\right)$.

Following the theory of $s$-numbers presented in $[11,13]$ and $[2,1]$, we introduce the notion of an $m$-quasi $s$-number sequence for $m$-multilinear bounded operators. For each $m \in \mathbf{N}$, a rule $s=\left(s_{n}\right): \mathcal{L}_{m}\left(X_{1} \times \cdots \times X_{m}, Y\right) \rightarrow[0, \infty)^{\mathbf{N}}$ assigning to every operator $T \in \mathcal{L}_{m}\left(X_{1} \times \cdots \times X_{m}, Y\right)$ a non-negative scalar sequence $\left(s_{n}(T)\right)$, it is called an $m$-quasi $s$-number sequence if the following conditions are satisfied:

(S1) Monotonicity: For every $T \in \mathcal{L}_{m}\left(X_{1} \times \cdots \times X_{m}, Y\right)$,

$$
\|T\|=s_{1}(T) \geq s_{2}(T) \geq \cdots \geq 0 .
$$

(S2) Additivity: For every $S, T \in \mathcal{L}_{m}\left(X_{1} \times \cdots \times X_{m}, Y\right)$,

$$
s_{k+n-1}(S+T) \leq s_{k}(S)+s_{n}(T) .
$$

(S3) Ideal-property: For every $T \in \mathcal{L}_{m}\left(X_{1} \times \cdots \times X_{m}, Y\right), S \in \mathcal{L}(Y, Z)$,

$$
s_{n}(S T) \leq\|S\| s_{n}(T) .
$$

(S4) Rank-property:

$$
\operatorname{rank}(T)<n \Rightarrow s_{n}(T)=0 .
$$

If $\left(s_{n}\right)$ is an $m$-quasi $s$-number sequence for each positive integer $m$, then $\left(s_{n}\right)$ is called a quasi $s$-number sequence. A quasi $s$-number sequence is called an $s$-number sequence provided it satisfies 
(S5) Norming property:

$$
s_{n}\left(I: \ell_{2}^{n} \rightarrow \ell_{2}^{n}\right)=1, \quad n \in \mathbf{N},
$$

where $I$ denotes the identity operator on the $n$-dimensional Hilbert space $\ell_{2}^{n}$.

For simplicity of notation and presentation, similarly as in the linear case, we do not indicate the involved Banach spaces and we write for short $s_{n}(T)$ instead of $s_{n}\left(T: X_{1} \times \cdots \times X_{m} \rightarrow Y\right)$.

We also need the following notions:

(J) An $m$-quasi $s$-number sequence $s=\left(s_{n}\right)$ is called injective if, given any metric injection $j \in \mathcal{L}(Y, Z)$, i.e., $\|j(y)\|=\|y\|$ for all $y \in Y, s_{n}(T)=s_{n}(j T)$ for all $T \in \mathcal{L}_{m}\left(X_{1} \times \cdots \times X_{m}, Y\right)$ and all $m$-tuples of Banach spaces $\left(X_{1}, \ldots, X_{m}\right)$.

(S) An $m$-quasi $s$-number sequence $s=\left(s_{n}\right)$ is called surjective if, given any metric surjections $Q_{j} \in \mathcal{L}\left(Y_{j}, X_{j}\right), 1 \leq j \leq m$, i.e., $Q_{j}\left(\stackrel{\circ}{U}_{Y_{j}}\right)=\stackrel{\circ}{U}_{X_{j}}$ for each $1 \leq j \leq m, s_{n}(T)=s_{n}\left(T\left(Q_{1} \times \cdots \times Q_{m}\right)\right)$ for all $T \in \mathcal{L}_{m}\left(X_{1} \times \cdots \times X_{m}, Y\right)$ and all Banach spaces $Y$, where $Q_{1} \times \cdots \times Q_{m}$ is the linear operator from $Y_{1} \times \cdots \times Y_{m}$ into $X_{1} \times \cdots \times X_{m}$ defined by

$Q_{1} \times \cdots \times Q_{m}\left(y_{1}, \ldots, y_{m}\right)=\left(Q_{1} y_{1}, \ldots, Q_{m} y_{m}\right), \quad\left(y_{1}, \ldots, y_{m}\right) \in Y_{1} \times \cdots \times Y_{m}$.

(JS) An $m$-quasi $s$-number sequence is called injective and surjective, if it satisfies $(\mathrm{J})$ and $(\mathrm{S})$.

(M) A quasi $s$-number sequence $s=\left(s_{n}\right)$ is called multiplicative if, for $S \in \mathcal{L}(Y, Z)$ and $T \in \mathcal{L}_{m}\left(X_{1} \times \cdots \times X_{m}, Y\right)$,

$$
s_{k+n-1}(S T) \leq s_{k}(S) s_{n}(T), \quad k, n \in \mathbf{N} .
$$

The paper is organized as follows. In Section 2 we investigate the measure of non-compactness of multilinear operators among Banach spaces. The main results of this section states that, up to universal constants, the measure of non-compactness of every multilinear operator $T$ is equivalent to the measure of non-compactness of its generalized adjoint (adjoint for short) operator $T^{\times}$of $T$. As a consequence we obtain a variant of Schauder's theorem for multilinear operators, which was first proved for the bilinear case by Ramanujan and Schock [15].

In Section 3 an $s$-sequence of approximation numbers of multilinear operators is studied. This sequence is used to provide a multilinear variant of a remarkable Carl's mixing property for any quasi $s$-number sequence of multilinear operators. The relationships between approximation numbers of multilinear operator and its generalized linear adjoint operator are also obtained. In particular we show that for every compact multilinear operator $T, a_{n}(T)=a_{n}\left(T^{\times}\right)$for each positive integer $n$.

Section 4 is devoted to an $s$-sequence $\left(d_{n}\right)$ of Kolmogorov numbers of multilinear operators. We show the fundamental properties of these numbers. Among others, we show that, as in the linear case, $\left(d_{n}\right)$ is the largest surjective multiplicative quasi $s$-number sequence.

In Section 5 we define variants of Gelfand's numbers $\left(c_{n}\right)$ of multilinear operators. It is proved that the Gelfand numbers is the largest injective multiplicative quasi $s$-number sequence and also that, in the multilinear case, variants of important relations between Gelfand and Kolmogorov numbers of an operator and its adjoint are true. Namely, we prove that $c_{n}\left(T^{\times}\right) \leq d_{n}(T)$ and $c_{n}(T)=d_{n}\left(T^{\times}\right)$are true for every multilinear operator $T$ and each positive integer $n$. In the last part of Section 
5 we investigate the relationships between variants of Gelfand's numbers. The main key here is the multilinear extension property of multilinear forms. Using famous Maurey's extension theorem for the bilinear forms, we show applications to the bilinear operators $T: X \times Y \rightarrow Z$, where $X$ and $Y$ are finite dimensional Banach spaces of type 2 .

\section{Measures of non-compactness of multilinear operators}

The present section is devoted to the relationships among the corresponding measure of non-compactness of multilinear operator and its adjoint. Let us recall that Schauder's well-known result states that an operator $T$ between Banach spaces is compact if, and only if, its adjoint, $T^{*}$, is compact. A slightly more general result says that the Kuratowski-Hausdorff measure of non-compactness of an operator $T$ is equivalent to the measure of non-compactness of its adjoint. Ramanujan and Schock studied in [15] ideals of bilinear operators between Banach spaces, including the ideal of bilinear compact operators, i.e., $T \in \mathcal{L}_{2}(X \times Y, Z)$ such that $T\left(U_{X} \times U_{Y}\right)$ is relatively compact in $Z$. Given $T \in \mathcal{L}_{2}(X \times Y, Z)$, they defined the adjoint linear $\operatorname{map} T^{\times}: Z^{*} \rightarrow \mathcal{L}_{2}(X \times Y)$ by

$$
T^{\times} z^{*}(x, y)=z^{*}(T(x, y)), \quad(x, y) \in X \times Y .
$$

Clearly $T^{\times}$is bounded operator, and $\|T\|=\left\|T^{\times}\right\|$. Ramanujan and Schock [15, Theorem 2.6] proved the analogues of Schauder's theorem which states that if $T \in$ $\mathcal{L}_{2}(X \times Y, Z)$, then $T$ is compact if, and only if $T^{\times}$is compact.

The mentioned results give rise to a question: whether the measures of noncompactness of a multilinear operator and its adjoint are equivalent? In the present section, we discuss this problem. We extend the classical well-known results for the linear operators to the case of multilinear operators.

Let us recall that if $A$ is a bounded subset of a metric space $\mathcal{X}$, the Kuratowski measure of non-compactness is defined by

$$
\alpha(A)=\inf \{\varepsilon>0 ; A \text { may be covered by finitely many sets of diameter } \leq \varepsilon\} ;
$$

the Hausdorff ball measure of non-compactness of $A$ is defined by

$$
\beta(A)=\inf \{\varepsilon>0 ; A \text { can be covered by finitely many balls of radius } \leq \varepsilon\} .
$$

It is easy to check that $\beta(A) \leq \alpha(A) \leq 2 \beta(A)$ for every bounded set.

Recall that in a Banach space $X$, a set $S$ is called an $\varepsilon$-net of $A$ if $A \subset S+\varepsilon U_{X}$. Thus the definition of $\beta$-measure in a Banach space is equivalent to the following:

$$
\beta(A)=\inf \{\varepsilon>0 ; A \text { has a finite } \varepsilon \text {-net }\} .
$$

Let $X_{1}, \ldots, X_{m}$ and $Y$ be Banach spaces. The Kuratowski, and the Hausdorff measure of non-compactness of $T \in \mathcal{L}_{m}\left(X_{1} \times \cdots \times X_{m}, Y\right)$ are defined by

$$
\gamma(T)=\alpha\left(T\left(U_{X_{1} \times \cdots \times X_{m}}\right)\right)
$$

and respectively,

$$
\widetilde{\gamma}(T)=\beta\left(T\left(U_{X_{1} \times \cdots \times X_{m}}\right)\right) .
$$

Since $T\left(U_{X_{1} \times \cdots \times U_{X_{m}}}\right) \subset\|T\| U_{Y}, \gamma(T) \leq\|T\|$. It is clear that $T$ is compact if, and only if, $\gamma(T)=0$. 
Throughout the paper the map $J: X_{1} \times \cdots \times X_{m} \rightarrow \mathcal{L}_{m}\left(X_{1} \times \cdots \times X_{m}\right)^{*}$ is defined by

$$
J x(B)=B x, \quad x \in X_{1} \times \cdots \times X_{m}, B \in \mathcal{L}_{m}\left(X_{1} \times \cdots \times X_{m}\right) .
$$

Clearly $J$ is a bounded $m$-linear operator. Given $T \in \mathcal{L}_{m}\left(X_{1} \times \cdots \times X_{m}, Y\right)$, we define the generalized adjoint (adjoint for short) operator $T^{\times}: Y^{*} \rightarrow \mathcal{L}_{m}\left(X_{1} \times \cdots \times X_{m}\right)$ by

$$
\left(T^{\times} y^{*}\right) x=y^{*}(T x), \quad y^{*} \in Y^{*}, x \in X_{1} \times \cdots \times X_{m} .
$$

For $B \in \mathcal{L}_{m}\left(X_{1} \times \cdots \times X_{m}\right)$ and $y \in Y$, by $B \otimes y$ we denote the $m$-linear operator

$$
B \otimes y=B(x) \cdot y, \quad x \in X_{1} \times \cdots \times X_{m} .
$$

Obviously $\operatorname{Im}(B \otimes y)$ is a linear subspace of $Y$ and $\operatorname{rank}(B \otimes y)=1$ provided $B \neq 0$ and $y \neq 0$. We also have

$$
(B \otimes y)^{\times}=\kappa_{Y}(y) \otimes B .
$$

We will need the following observation.

Lemma 2.1. Let $m \geq 2$ and let $X_{1}, \ldots, X_{m}, Y$ be Banach spaces. Then for every operator $T \in \mathcal{L}_{m}\left(X_{1} \times \cdots \times X_{m}, Y\right)$,

$$
\left(T^{\times}\right)^{*} J=\kappa_{Y} T \text {. }
$$

Proof. Let $x \in X_{1} \times \cdots \times X_{m}$ and $y^{*} \in Y^{*}$. Then we have

$$
\left\langle y^{*},\left(T^{\times}\right)^{*} J(x)\right\rangle=\left\langle T^{\times} y^{*}, J(x)\right\rangle=\left(y^{*} \circ T\right)(x)=\left\langle T x, y^{*}\right\rangle=\left\langle y^{*}, \kappa_{Y} T(x)\right\rangle,
$$

and this yields the required equality.

A well-known result about linear operators states that for every operator $T: X \rightarrow$ $Y$ between Banach spaces $X$ and $Y$, we have ([4, Theorem 2.9])

$$
\gamma(T) \leq \widetilde{\gamma}\left(T^{*}\right) \quad \text { and } \quad \gamma\left(T^{*}\right) \leq \widetilde{\gamma}(T) .
$$

We will now prove analogous results in the multilinear case.

Theorem 2.1. Let $m \geq 2$ and let $X_{1}, \ldots, X_{m}, Y$ be Banach spaces. Then the following estimates hold for every $T \in \mathcal{L}_{m}\left(X_{1} \times \cdots \times X_{m}, Y\right)$ :

$$
\gamma(T) \leq \widetilde{\gamma}\left(T^{\times}\right) \quad \text { and } \quad \gamma\left(T^{\times}\right) \leq \widetilde{\gamma}(T)
$$

Proof. We claim that $\gamma(T) \leq \widetilde{\gamma}\left(T^{\times}\right)$. Applying the mentioned above result about linear operators, we have

$$
\gamma\left(\left(T^{\times}\right)^{*}\right) \leq \widetilde{\gamma}\left(T^{\times}\right) .
$$

Since $\kappa_{Y}$ is an isometry and $\|J\|=1$, the above inequality in combination with Lemma 2.1 yields

$$
\begin{aligned}
\gamma(T) & =\alpha\left(T\left(U_{X_{1} \times \cdots \times X_{m}}\right)\right)=\alpha\left(\kappa_{Y} T\left(U_{X_{1} \times \cdots \times X_{m}}\right)\right) \\
& =\alpha\left(\left(T^{\times}\right)^{*} J\left(U_{X_{1} \times \cdots \times X_{m}}\right)\right) \leq \gamma\left(\left(T^{\times}\right)^{*}\right) \alpha\left(J\left(U_{X_{1} \times \cdots \times X_{m}}\right)\right) \\
& \leq \widetilde{\gamma}\left(T^{\times}\right) \alpha\left(\|J\| U_{Z^{*}}\right) \leq \widetilde{\gamma}\left(T^{\times}\right)
\end{aligned}
$$

where $Z:=\mathcal{L}_{m}\left(X_{1} \times \cdots \times X_{m}\right)$, and this completes the proof of the claim.

Put $k:=\gamma(T)$, and let $S \subset Y^{*}$ be any set with $\operatorname{diam}(S) \leq d, d>0$. To prove the second inequality we only need to show that $T^{\times}(S)$ can be covered by finitely 
many sets with diameter less or equal than $k d$. To do this fix $\varepsilon>0$. Then there exist $y_{1}, \ldots, y_{n} \in Y$, such that

$$
T\left(U_{X_{1} \times \cdots \times X_{m}}\right) \subset \bigcup_{j=1}^{n}\left(y_{j}+r U_{Y}\right),
$$

where $r=\gamma(T)+\varepsilon / 2 d$.

For each $1 \leq j \leq n$, we define the set

$$
\left\{y^{*}\left(y_{j}\right) ; y^{*} \in S\right\} \subset \mathbf{K} .
$$

Since $S$ is bounded, the sets are relatively compact. Without loss of generality, we may assume that $\mathbf{K}=\mathbf{R}$. Thus, for each $1 \leq j \leq n$, the above set may be covered by closed intervals $I_{j, 1}, \ldots, I_{j, m(j)}$ with length less or equal than $\varepsilon / 2$.

Let $p=\left(p_{1}, \ldots, p_{n}\right)$, where $p_{j} \in\{1,2, \ldots, m(j)\}$, and let us set

$$
E_{p}:=\left\{y^{*} \in S ;\left\langle y, y^{*}\right\rangle \in I_{j, p_{j}}, 1 \leq j \leq n\right\} .
$$

Clearly $T^{\times}(S) \subset \bigcup_{p} T^{\times}\left(E_{p}\right)$ with a finite union. We shall show that $\operatorname{diam}\left(T^{\times}\left(E_{p}\right)\right)<$ $\gamma(T) d+\varepsilon$ for all $p$. To show this fix $p$ and take $y_{1}^{*}, y_{2}^{*} \in E_{p}$. Then

$$
\begin{aligned}
\left\|T^{\times} y_{1}^{*}-T^{\times} y_{2}^{*}\right\| & =\sup \left\{\left|\left\langle T x, y_{1}^{*}-y_{2}^{*}\right\rangle\right| ; x \in U_{X_{1} \times \cdots \times X_{m}}\right\} \\
& =\sup \left\{\left|\left\langle y, y_{1}^{*}-y_{2}^{*}\right\rangle\right| ; y \in T\left(U_{X_{1} \times \cdots \times X_{m}}\right)\right\} .
\end{aligned}
$$

Now, observe that for every $y \in T\left(U_{X_{1} \times \cdots \times X_{m}}\right)$ there exists $1 \leq j \leq n$ such that $y \in y_{j}+r U_{Y}$. Since $y_{1}^{*}, y_{2}^{*} \in E_{p},\left|\left\langle y_{j}, y_{1}^{*}-y_{2}^{*}\right\rangle\right|<\varepsilon / 2$. This implies, by $\left\|y_{1}^{*}-y_{2}^{*}\right\| \leq d$ and $\left\|y-y_{j}\right\| \leq \gamma(T)+\varepsilon / 2 d$,

$$
\left|\left\langle y-y_{j}, y_{1}^{*}-y_{2}^{*}\right\rangle\right| \leq\left\|y_{1}^{*}-y_{2}^{*}\right\|\left\|y-y_{j}\right\| \leq \gamma(T) d+\varepsilon / 2 .
$$

Hence

$$
\left|\left\langle y, y_{1}^{*}-y_{2}^{*}\right\rangle\right| \leq\left|\left\langle y-y_{j}, y_{1}^{*}-y_{2}^{*}\right\rangle\right|+\left|\left\langle y_{j}, y_{1}^{*}-y_{2}^{*}\right\rangle\right|<\gamma(T) d+\varepsilon .
$$

The combination of the above estimates yields

$$
\left|\left(T^{\times} y_{1}^{*}-T^{\times} y_{2}^{*}\right) x\right|<\gamma(T) d+\varepsilon
$$

for all $x \in U_{X_{1} \times \cdots \times X_{m}}$. Since $\varepsilon>0$ was arbitrary,

$$
\left\|T^{\times} y_{1}^{*}-T^{\times} y_{2}^{*}\right\| \leq \gamma(T) d
$$

and this completes the proof.

Corollary 2.1. Let $m \geq 2$ and let $X_{1}, \ldots, X_{m}, Y$ be Banach spaces. Then the following estimates hold for every operator $T \in \mathcal{L}_{m}\left(X_{1} \times \cdots \times X_{m}, Y\right)$ :

$$
\frac{1}{2} \gamma(T) \leq \gamma\left(T^{\times}\right) \leq 2 \gamma(T) \quad \text { and } \quad \frac{1}{2} \widetilde{\gamma}(T) \leq \widetilde{\gamma}\left(T^{\times}\right) \leq 2 \widetilde{\gamma}(T) .
$$

As a consequence, we obtain Schauder's theorem for multilinear operators, proved for the bilinear case by Ramanujan and Schock [15, Theorem 2.6].

Corollary 2.2. Let $m \geq 2$ and let $X_{1}, \ldots, X_{m}, Y$ be Banach spaces. Then $T \in \mathcal{L}_{m}\left(X_{1} \times \cdots \times X_{m}, Y\right)$ is compact if, and only if, $T^{\times}$is compact. 


\section{Approximation numbers of multilinear operators}

One of the most important examples of an $s$-number sequence is the sequence $\left(a_{n}\right)$ of approximation numbers. Let us recall that for any operator $T \in \mathcal{L}(X, Y)$ and $n \in \mathbf{N}$ the $n$-th approximation number $a_{n}(T)$ is given by

$$
a_{n}(T):=\inf \{\|T-A\| ; A \in \mathcal{L}(X, Y), \operatorname{rank}(A)<n\}
$$

(cf. $[11,13])$.

Let $X_{1}, \ldots, X_{m}$ and $Y$ be Banach spaces and let $T \in \mathcal{L}_{m}\left(X_{1} \times \cdots \times X_{m}, Y\right)$. We denote the image of $T$ by $\operatorname{Im}(T)$. Since it is not generally the case that $\operatorname{Im}(T)$ is a linear subspace of $Y$, we define $\operatorname{rank}(T)$ as the dimension of $[\operatorname{Im}(T)]$, where $[E]$ denotes the linear span of a subset $E$ in a vector space $V$.

We define the $n$-th approximation number $a_{n}(T)$ of any multilinear operator $T \in \mathcal{L}_{m}\left(X_{1} \times \cdots \times X_{m}, Y\right)$ by

$$
a_{n}(T):=\inf \left\{\|T-A\| ; A \in \mathcal{L}_{m}\left(X_{1} \times \cdots \times X_{m}, Y\right), \operatorname{rank}(A)<n\right\} .
$$

It is easy to check that $\left(a_{n}\right)$ is an $s$-number sequence.

We will state below some fundamental properties of approximation numbers of multilinear maps, and for the sake of completeness we include proofs. It should be pointed out that Carl [1] was among the first to discover the mixing multiplicativity property of bounded linear operators and used it to study $s$-numbers of bounded linear operators among Banach spaces.

Proposition 3.1. Assume $\left(s_{n}\right): \mathcal{L}_{m}\left(X_{1} \times \cdots \times X_{m}, Y\right) \rightarrow[0, \infty)^{\mathbf{N}}$ is a sequence which satisfies the monotonicity $(S 1)$, the additivity $(S 2)$ and the rank property $(S 4)$.

(i) An approximation sequence $\left(a_{n}\right)$ is the largest $\left(s_{n}\right)$ sequence which satisfies

$$
s_{n}(T) \leq a_{n}(T), \quad n \in \mathbf{N} .
$$

(ii) If $\left(s_{n}\right)$ is a quasi $s$-number sequence, then it has the mixing multiplicativity property, i.e., for all $S \in \mathcal{L}(Y, Z), T \in \mathcal{L}_{m}\left(X_{1} \times \cdots \times X_{m}, Y\right)$ and all $k, n \in \mathbf{N}$ we have

$$
s_{k+n-1}(S T) \leq s_{k}(S) a_{n}(T) \text { and } s_{k+n-1}(S T) \leq a_{k}(S) s_{n}(T) .
$$

Proof. (i) Let $T \in \mathcal{L}_{m}\left(X_{1} \times \cdots \times X_{m}, Y\right)$. Then for any $A \in \mathcal{L}_{m}\left(X_{1} \times \cdots \times X_{m}, Y\right)$ with $\operatorname{rank}(A)<n$, we have

$$
s_{n}(T) \leq\|T-A\|+s_{n}(A)=\|T-A\|
$$

and this yields $s_{n}(T) \leq a_{n}(T)$.

(ii) Let $A \in \mathcal{L}\left(X_{1} \times \cdots \times X_{m}, Y\right)$ be an operator with $\operatorname{rank}(A)<n$. Since $\operatorname{rank}(S A)<n$, it follows, by properties $(\mathrm{S} 1),(\mathrm{S} 2)$ and $(\mathrm{S} 4)$, that

$$
\begin{aligned}
s_{k+n-1}(S T) & \leq s_{k+n-1}\left((S(T-A)+S A) \leq s_{k}(S(T-A))+s_{n}(S A)\right. \\
& =s_{k}(S(T-A)) \leq s_{k}(S)\|T-A\|
\end{aligned}
$$

and this completes the proof of the first inequality. The proof of the second inequality is very similar to the first one and so it will be omitted.

Below we state and prove certain relationships between approximation numbers of an $m$-linear bounded operator $T$ and its generalized adjoint operator $T^{\times}$. We need the following. 
Lemma 3.1. Let $m \geq 2$ and $X_{1}, \ldots, X_{m}, Y$ be Banach spaces. If $T \in \mathcal{L}_{m}\left(X_{1} \times\right.$ $\left.\cdots \times X_{m}, Y\right)$ has finite rank, then

$$
\operatorname{rank}(T)=\operatorname{rank}\left(T^{\times}\right) .
$$

Proof. Let $n:=\operatorname{rank}(T)$. Then $\operatorname{dim}(V)=n$, where $V=[\operatorname{Im}(T)] \subset Y$. We use Auerbach's lemma which states, that there are unit vectors $v_{1}, \ldots, v_{n} \in V$ and unit vectors $v_{1}^{*}, \ldots, v_{n}^{*} \in V^{*}$, such that

$$
\left\langle v_{i}, v_{j}^{*}\right\rangle=\delta_{i j}, \quad 1 \leq i, j \leq n .
$$

Obviously, $\left\{v_{1}, \ldots, v_{n}\right\}$ forms a basis for $V$, and $\left\{v_{1}^{*}, \ldots, v_{n}^{*}\right\}$ is a dual basis for $V^{*}$. By the Hahn-Banach theorem, there exists $y_{j}^{*} \in Y^{*}$, such that $y_{j}^{*}$ is $v_{j}^{*}$ on $V$.

Since $T x \in V$ for all $x \in X_{1} \times \cdots \times X_{m}$,

$$
T x=\sum_{j=1}^{n} v_{j}^{*}(T x) v_{j}=\sum_{j=1}^{n}\left(T^{\times} y_{j}^{*}\right)(x) v_{j}=\sum_{j=1}^{n}\left(T^{\times} y_{j}^{*} \otimes v_{j}\right)(x) ;
$$

that is, $T=\sum_{j=1}^{n} T^{\times} y_{j}^{*} \otimes v_{j}$. Consequently,

$$
T^{\times}=\sum_{j=1}^{n} \kappa_{Y}\left(v_{j}\right) \otimes B_{j},
$$

where $B_{j}:=T^{\times} y_{j}^{*} \otimes v_{j}$ for each $1 \leq j \leq n$. Since $\kappa_{Y}\left(v_{j}\right) \in Y^{* *} \neq 0$ and $B_{j} \in$ $\mathcal{L}_{m}\left(X_{1} \times \cdots \times X_{m}\right)$ with $\operatorname{rank}\left(B_{j}\right)=1, \operatorname{rank}\left(T^{\times}\right) \leq n$. We claim that $\operatorname{rank}\left(T^{\times}\right)=n$. To see this we only need to show that $\left\{B_{1}, \ldots, B_{n}\right\}$ is a linearly independent set in $\mathcal{L}_{m}\left(X_{1} \times \cdots \times X_{m}\right)$. Let $\lambda_{1}, \ldots, \lambda_{n} \in \mathbf{K}$ be such that

$$
\sum_{j=1}^{n} \lambda_{j} B_{j}=0
$$

that is, $\sum_{j=1}^{n} \lambda_{j}\left(T^{\times} y_{j}^{*} \otimes v_{j}\right)(x)=\sum_{j=1}^{n} \lambda_{j} v_{j}^{*}(T x) v_{j}=0$, for all $x \in X_{1} \times \cdots \times X_{m}$. This implies that for all $v \in V$ we get

$$
\sum_{j=1}^{n} \lambda_{j} v_{j}^{*}(v) v_{j}=0
$$

Since $v_{i}^{*}\left(v_{j}\right)=\delta_{i j}$ for all $1 \leq i, j \leq n$, it follows that $\lambda_{j}=0$ for each $1 \leq j \leq n$. This completes the proof of the claim.

Below we will state and prove certain relationships between approximation numbers of an $m$-linear bounded operator $T$ and its generalized adjoint operator $T^{\times}$.

Proposition 3.2. For every operator $\mathcal{L}_{m}\left(X_{1} \times \cdots \times X_{m}, Y\right)$ we have

$$
a_{n}\left(T^{\times}\right) \leq a_{n}(T), \quad n \in \mathbf{N} .
$$

Proof. Given $\varepsilon>0$, there exists $A \in \mathcal{L}_{m}\left(X_{1} \times \cdots \times X_{m}, Y\right)$ with $\operatorname{rank}(A)<n$, such that

$$
\|T-A\| \leq(1+\varepsilon) a_{n}(T) .
$$

An application of Lemma 3.1 ensures that $\operatorname{rank}\left(A^{\times}\right)<n$. Thus combining with

$$
\left\|T^{\times}-A^{\times}\right\|=\|T-A\| \leq(1+\varepsilon) a_{n}(T),
$$

we conclude that

$$
a_{n}\left(T^{\times}\right) \leq(1+\varepsilon) a_{n}(T)
$$


Since $\varepsilon>0$ is arbitrary, the result follows.

Proposition 3.3. Let $Y$ be a Banach space such that there exists a linear projection $P$ of unit norm from $Y^{* *}$ onto $\kappa_{Y}(Y)$. Then for every $T \in \mathcal{L}_{m}\left(X_{1} \times \cdots \times X_{m}, Y\right)$

$$
a_{n}\left(T^{\times}\right)=a_{n}(T), \quad n \in \mathbf{N} .
$$

Proof. Fix $\varepsilon>0$. Then, there exists a linear operator $S: \mathcal{L}_{m}\left(X_{1} \times \cdots \times X_{m}, Y\right)^{*} \rightarrow$ $Y^{* *}$ with $\operatorname{rank}(S)<n$, such that

$$
\left\|\left(T^{\times}\right)^{*}-S\right\|<a_{n}\left(\left(T^{\times}\right)^{*}\right)+\varepsilon .
$$

Let $A=P S J$, where $J: X_{1} \times \cdots \times X_{m} \rightarrow \mathcal{L}_{m}\left(X_{1} \times \cdots \times X_{m}\right)^{*}$ is given by $J x(B)=B(x)$ for all $x \in X_{1} \times \cdots \times X_{m}$ and $B \in \mathcal{L}_{m}\left(X_{1} \times \cdots \times X_{m}\right)$. Then $A \in \mathcal{L}_{m}\left(X_{1} \times \cdots \times X_{m}, Y\right)$ with $\operatorname{rank}(A)<n$. Our hypothesis $\|P\|=1$ in combination with $\|J\|=1$ yields

$$
\|T-S\|=\left\|P\left(T^{\times}\right)^{*} J-P S J\right\| \leq\left\|\left(T^{\times}\right)^{*}-S\right\|<a_{n}\left(\left(T^{\times}\right)^{*}\right)+\varepsilon .
$$

Since $\varepsilon$ is arbitrary, we conclude that

$$
a_{n}(T) \leq a_{n}\left(\left(T^{\times}\right)^{*}\right) \leq a_{n}\left(T^{\times}\right) .
$$

This completes the proof by Proposition 3.2.

Applications of above results will be shown. Note that Edmunds and Tylli [5] proved that, for any operator $T \in \mathcal{L}(E, F)$ between Banach spaces $E$ and $F$, the following estimate holds

$$
a_{n}(T) \leq a_{n}\left(T^{* *}\right)+2 \gamma(T), \quad n \in \mathbf{N} .
$$

In the multilinear case, we have the following result.

Theorem 3.1. For every operator $T \in \mathcal{L}_{m}\left(X_{1} \times \cdots \times X_{m}, Y\right)$ we have

$$
a_{n}(T) \leq a_{n}\left(\left(T^{\times}\right)^{*}\right)+2 \widetilde{\gamma}(T), \quad n \in \mathbf{N} .
$$

Proof. Let $\varepsilon>0$ and $\lambda>\widetilde{\gamma}(T)$. Then there exists a linear operator $A: \mathcal{L}_{m}\left(X_{1} \times\right.$ $\left.\cdots \times X_{m}\right)^{*} \rightarrow Y^{* *}$ with $\operatorname{rank}(A)<n$ such that

$$
\left\|\left(T^{\times}\right)^{*}-A\right\|<a_{n}\left(\left(T^{\times}\right)^{*}\right)+\varepsilon .
$$

Let $y_{1}, \ldots, y_{k} \in Y$ with $T\left(U_{X_{1} \times \cdots \times X_{m}}\right) \subset\left\{y_{1}, \ldots, y_{k}\right\}+\lambda U_{Y}$. Let $M$ be the linear span of $\operatorname{Im}(A) \cup\left\{\kappa_{Y}\left(z_{j}\right) ; 1 \leq j \leq k\right\}$. By the principle of local reflexivity, there exists $R: M \rightarrow Y$ such that $\|R\| \leq 1+\varepsilon$ and $R \kappa_{Y}\left(y_{j}\right)=y_{j}$, for each $1 \leq j \leq k$.

Define $S:=R A J \in \mathcal{L}_{m}\left(X_{1} \times \cdots \times X_{m}, Y\right)$. Then $\operatorname{rank}(S) \leq n$. For every $x \in U_{X_{1} \times \cdots \times X_{m}}$, we choose $y_{j}$ with $\left\|T x-y_{j}\right\| \leq \lambda$ for some $1 \leq j \leq k$. Since $\left(T^{\times}\right)^{*} J=\kappa_{Y} T$

$$
\begin{aligned}
\|T x-S x\| & \leq\left\|T x-y_{j}\right\|+\left\|y_{j}-S x\right\| \leq \lambda+\left\|R \kappa_{Y} y_{j}-R A J x\right\| \\
& \leq \lambda+(1+\varepsilon)\left(\left\|\kappa_{Y} y_{j}-\kappa_{Y} T x\right\|+\left\|\left(T^{\times}\right)^{*} J x-A J x\right\|\right) \\
& \leq \lambda+(1+\varepsilon)\left(\lambda+a_{n}\left(\left(T^{\times}\right)^{*}\right)+\varepsilon\right) .
\end{aligned}
$$

Since $\varepsilon>0$ and $\lambda>\widetilde{\gamma}(T)$ are arbitrary, we obtain the required estimate.

As an application of the above results, we obtain the following multilinear variants of the well-known results in the linear case (see [5]).

Corollary 3.1. If $T \in \mathcal{L}_{m}\left(X_{1} \times \cdots \times X_{m}, Y\right)$ is a compact operator, then

$$
a_{n}(T)=a_{n}\left(T^{\times}\right), \quad n \in \mathbf{N} .
$$


Proof. Since $T^{\times}$is a linear operator among Banach spaces, $a_{n}\left(\left(T^{\times}\right)^{*}\right) \leq a_{n}\left(T^{\times}\right)$. If $T$ is compact, then $\widetilde{\gamma}(T)=0$ and so

$$
a_{n}(T) \leq a_{n}\left(\left(T^{\times}\right)^{*}\right) \leq a_{n}\left(T^{\times}\right) \leq a_{n}(T),
$$

by Theorem 3.1 and Proposition 3.2.

Corollary 3.2. For every operator $T \in \mathcal{L}_{m}\left(X_{1} \times \cdots \times X_{m}, Y\right)$ we have,

$$
a_{n}(T) \leq 5 a_{n}\left(T^{\times}\right), \quad n \in \mathbf{N} .
$$

Proof. Fix $n \in \mathbf{N}$. Let $S_{n}: Y^{*} \rightarrow \mathcal{L}_{m}\left(X_{1} \times \cdots \times X_{m}\right)$ be an arbitrary linear operator with $\operatorname{rank}\left(S_{n}\right)<n$. Then

$$
\widetilde{\gamma}\left(T^{\times}\right)=\widetilde{\gamma}\left(T^{\times}-S_{n}\right) \leq\left\|T^{\times}-S_{n}\right\| .
$$

This shows that $\widetilde{\gamma}\left(T^{\times}\right) \leq a_{n}\left(T^{\times}\right)$and so

$$
a_{n}(T) \leq a_{n}\left(\left(T^{\times}\right)^{*}\right)+2 \widetilde{\gamma}(T) \leq a_{n}\left(T^{\times}\right)+4 \widetilde{\gamma}\left(T^{\times}\right) \leq 5 a_{n}\left(T^{\times}\right),
$$

and this completes the proof.

\section{Kolmogorov numbers of multilinear operators}

Since for every multilinear operator $T \in \mathcal{L}_{m}\left(X_{1} \times \cdots \times X_{m}, Y\right)$ and any $m$ quasi $s$-sequence $\left(s_{n}\right)$, the sequence $\left(s_{n}(T)\right)$ is non-increasing and bounded below by 0 , so it has a limit. In particular the measure of non-approximability, $a(T):=$ $\lim _{n \rightarrow \infty} a_{n}(T)$ exists. If $a(T)=0$, then clearly $T$ is approximable (i.e., there exists a sequence $\left(A_{n}\right)$ of finite dimensional operators $A_{n} \in \mathcal{L}_{m}\left(X_{1} \times \cdots \times X_{m}, Y\right)$, such that $\left.\lim _{n \rightarrow \infty}\left\|T-A_{n}\right\|=0\right)$ and so it is compact. It is well-known that in general the converse is false. Roughly speaking, the quantity $a(T)$ is not a useful measure of the deviation of an operator $T$ from compactness.

Similarly to the linear case, if the target space $Y$ has the approximation property, that is, given any compact subset $K$ of $Y$ and every $\varepsilon>0$, there is $a$ finite dimensional linear map $S: Y \rightarrow Y$, such that $\|S y-y\|<\varepsilon$ for all $y \in K$, then, it is easy to see that any compact $m$-linear operator $T: X_{1} \times \cdots \times X_{m} \rightarrow Y$ can be approximated arbitrarily and closely by finite-dimensional $m$-linear operators. In consequence, $T \in \mathcal{L}_{m}\left(X_{1} \times \cdots \times X_{m}, Y\right)$ is compact if, and only if, $a(T)=0$. We will use below this simple fact without any further references.

The quantity which is a useful measure of the deviation of change linear operator from compactness is connected with the Kolmogorov numbers.

Following the linear case, we define the $n$-th Kolgomorov number $d_{n}(T)$ of an operator $T \in \mathcal{L}_{m}\left(X_{1} \times \cdots \times X_{m}, Y\right)$ by

$$
d_{n}(T)=\inf \left\{\varepsilon>0 ; T\left(U_{X_{1} \times \cdots \times X_{m}}\right) \subset N_{\varepsilon}+\varepsilon U_{Y}, N_{\varepsilon} \subset Y, \operatorname{dim}\left(N_{\varepsilon}\right)<n\right\} .
$$

Clearly that $T \in \mathcal{L}_{m}\left(X_{1} \times \cdots \times X_{m}, Y\right)$ is a compact operator if and only if

$$
d(T):=\lim _{n \rightarrow \infty} d_{n}(T)=0 .
$$

It is also obvious that $d_{n}(T)=0$ provided $\operatorname{rank}(T)<n$.

Since in the above definition of $d_{n}(T)$ we may replace closed unit balls $U_{X_{1} \times \cdots \times X_{m}}$ or $U_{Y}$ by the corresponding open unit balls $\stackrel{\circ}{U}_{X_{1} \times \cdots \times X_{m}}$ or $\stackrel{\circ}{U}_{Y}$, it may be shown, 
similarly as in the linear case, that the following multilinear variant of Pietsch's formula holds (see $[12,1]$ )

$$
d_{n}(T)=\inf \left\{\left\|Q_{N}^{Y} T\right\| ; N \subset Y, \operatorname{dim}(N)<n\right\} .
$$

This formula easily gives $\left(d_{n}\right)$ is an $s$-number sequence, and so Proposition 3.1 implies that

$$
d_{n}(T) \leq a_{n}(T), \quad n \in \mathbf{N} .
$$

Note also that $\left(d_{n}\right)$ is a surjective quasi $s$-number sequence. We next present properties of Kolmogorov numbers similar to the linear case. To do this we need some further definition.

For a given $m \in \mathbf{N}$ and $i=1, \ldots, m$, let $X_{i}$ be a Banach space. We say that the Banach space $X_{1} \times \cdots \times X_{m}$ has the multilinear metric lifting property if, for every $\varepsilon>0$ and every bounded $m$-linear operator $T$ from $X_{1} \times \cdots \times X_{m}$ to any quotient space $Y / N$, there is $\widetilde{T} \in \mathcal{L}_{m}\left(X_{1} \times \cdots \times X_{m}, Y\right)$, such that

$$
T=Q_{N}^{Y} \widetilde{T} \text { and }\|\widetilde{T}\| \leq(1+\varepsilon)\|T\| .
$$

Proposition 4.1. Let $X_{1}, \ldots, X_{m}$ and $Y$ be Banach spaces. If $X_{1} \times \cdots \times X_{m}$ has the multilinear metric lifting property, then for every $T \in \mathcal{L}_{m}\left(X_{1} \times \cdots \times X_{m}, Y\right)$,

$$
d_{n}(T)=a_{n}(T), \quad n \in \mathbf{N} .
$$

Proof. Since $d_{n}(T) \leq a_{n}(T)$ for each $n$, we need to show the reverse inequality. Fix $\varepsilon>0$. Then there exists a subspace $N \subset Y$, such that $\operatorname{dim}(N)<n$ and $\left\|Q_{N}^{Y} T\right\|<d_{n}(T)+\varepsilon$. Our hypothesis yields that there exists $\widetilde{T} \in \mathcal{L}_{m}\left(X_{1} \times \cdots \times X_{m}, Y\right)$, such that

$$
Q_{N}^{Y} T=Q_{N}^{Y} \widetilde{T} \text { and }\|\widetilde{T}\| \leq(1+\varepsilon)\left\|Q_{N}^{Y} T\right\| .
$$

For $A:=T-\widetilde{T}$, we have $Q_{N}^{Y} A=0$. This implies $[\operatorname{Im}(T)] \subset N$, and so $\operatorname{rank}(S) \leq$ $\operatorname{dim}(N)<n$. In consequence

$$
a_{n}(T) \leq\|T-A\|=\|\widetilde{T}\| \leq(1+\varepsilon)\left\|Q_{N}^{Y} T\right\|<(1+\varepsilon)\left(d_{n}(T)+\varepsilon\right) .
$$

Since $\varepsilon>0$ is arbitrary, $a_{n}(T) \leq d_{n}(T)$ and so the proof is complete.

By the similar proof as in the linear case, one can see that $\ell_{1}\left(\Gamma_{1}\right) \times \cdots \times \ell_{1}\left(\Gamma_{m}\right)$ has the multilinear metric lifting property for each $m \geq 2$. Here, as usual, $\ell_{1}(\Gamma)$ is the Banach space of summable number families $\left\{\lambda_{\gamma}\right\}_{\gamma \in \Gamma}$ over an arbitrary index set.

It is well-known (see [11]) that in the linear case the Kolmogorov numbers $\left(d_{n}(T)\right)$ of every operator $T: X \rightarrow Y$ between Banach spaces $X$ and $Y$ may be characterized by the approximation numbers as follows

$$
d_{n}(T)=a_{n}\left(T Q_{1}\right)
$$

for each $n \in \mathbf{N}$, where $Q_{1}$ is the canonical metric surjection from $\ell_{1}\left(U_{X}\right)$ onto $X$, defined by

$$
Q_{1}\left(\left\{\lambda_{x}\right\}\right)=\sum_{x \in U_{X}} \lambda_{x} x, \quad\left\{\lambda_{x}\right\} \in \ell_{1}\left(U_{X}\right) .
$$

In the multilinear case, we have the following analogous result.

Theorem 4.1. Let $T \in \mathcal{L}_{m}\left(X_{1} \times \cdots \times X_{m}, Y\right)$ be an $m$-linear operator between Banach spaces, and let $Q:=Q_{1} \times \cdots \times Q_{j}$ where $Q_{j}$ is the canonical metric surjection from $\ell_{1}\left(U_{X_{j}}\right)$ onto $X_{j}$ for each $1 \leq j \leq m$. Then

$$
d_{n}(T)=a_{n}(T Q), \quad n \in \mathbf{N} .
$$


Proof. Since $\ell_{1}\left(U_{X_{1}}\right) \times \cdots \times \ell_{1}\left(U_{X_{m}}\right)$ has the multilinear metric lifting property, it follows from Proposition 4.1 that for each $n \in \mathbf{N}$,

$$
d_{n}(T Q)=a_{n}(T Q) .
$$

As noted, $\left(d_{n}\right)$ is a surjective $s$-number sequence, and this gives the required equality.

Note that for any surjective quasi $s$-number sequence the following estimate holds for all $S \in \mathcal{L}(Y, Z)$ and all $T \in \mathcal{L}_{m}\left(X_{1} \times \cdots \times X_{m}, Y\right)$,

$$
s_{k+n-1}(S T) \leq s_{k}(S) d_{n}(T), \quad k, n \in \mathbf{N} .
$$

The proof is similar to the linear case proof (see [1]). In fact, let $Q_{j}$ be the metric surjection from $\ell_{1}\left(U_{X_{j}}\right)$ onto $X_{j}$ for each $1 \leq j \leq m$. Then combining Theorem 4.1 with the multilinear multiplicativity property $(\mathrm{M})$, yields

$$
s_{k+n-1}(S T)=s_{k+n-1}(S T Q) \leq s_{k}(S) s_{n}(T Q) \leq s_{k}(S) a_{n}(T Q)=s_{k}(S) d_{n}(T) .
$$

An immediate consequence of the above fact is a multilinear variant of the wellknown result for the linear case, that the sequence $\left(d_{n}\right)$ of the Kolmogorov numbers is the largest surjective quasi $s$-number sequence which satisfies the multiplicativity property $(\mathrm{M})$ :

$$
d_{k+n-1}(S T) \leq d_{k}(S) d_{n}(T), \quad S \in \mathcal{L}(Y, Z), \quad T \in \mathcal{L}_{m}\left(X_{1} \times \cdots \times X_{m}, Y\right) .
$$

\section{Gelfand numbers of mulitilinear operators}

In the theory of $s$-numbers of linear operators, the Gelfand numbers play an important role. There are many equivalent definitions of Gelfand numbers; recall that the usual $n$-th Gelfand number $c_{n}(T)$ of an operator $T \in \mathcal{L}(X, Y)$ acting between arbitrary Banach spaces $X$ and $Y$ is defined to be the infimum of all $\varepsilon>0$, such that there are functionals $x_{i}^{*} \in X^{*}, 1 \leq i \leq k<n$, which admit an estimate

$$
\|T x\| \leq \sup _{1 \leq i \leq k}\left|\left\langle x, x_{i}^{*}\right\rangle\right|+\varepsilon\|x\|, \quad x \in X .
$$

It is well-known that in the linear case $\left(c_{n}\right)$ is an $s$-number sequence. For the basic facts about these numbers, which are given below, we refer to the books of Pietsch $[11,13]$.

In the multilinear case we make the following definition of Gelfand numbers; the $n$-th Gelfand number $c_{n}(T)$ of an operator $T \in \mathcal{L}_{m}\left(X_{1} \times \cdots \times X_{m}, Y\right)$ is defined by

$$
c_{n}(T)=a_{n}\left(\kappa_{Y} T\right) .
$$

This definition is motivated by the fact that the above formula holds in the case of linear operators, and many interesting applications may be found. Obviously $\left(c_{n}\right)$ is an $s$-number sequence by the fact that $\left(a_{n}\right)$ is an $s$-number sequence. Clearly we have $c_{n}(T) \leq a_{n}(T)$ for each $n \in \mathbf{N}$. In the case when $Y$ is a Banach space with the metric extension property (i.e., every operator $S \in \mathcal{L}(X, Y)$ from every Banach space $X$ to $Y$ can be extended to any Banach space $\widetilde{X}$ containing $X$ as a subspace, where the extension $\widetilde{T} \in \mathcal{L}(\widetilde{X}, Y)$ with $\|\widetilde{T}\|=\|T\|)$, then $c_{n}(T)=a_{n}(T)$.

We collect some properties of the Gelfand numbers $\left(c_{n}\right)$ for the multilinear case. The following result may be easily verified: If $Y$ is a Banach space with the metric 
extension property, $X_{1}, \ldots, X_{m}$ arbitrary Banach spaces and $T \in \mathcal{L}_{m}\left(X_{1} \times \cdots \times\right.$ $\left.X_{m}, Y\right)$, then

$$
c_{n}(T)=a_{n}(T), \quad n \in \mathbf{N} .
$$

Since any $\ell_{\infty}(\Gamma)$-space has the metric extension property, the above result immediately implies that $\left(c_{n}\right)$ is an injective $s$-number sequence.

The sequence $\left(c_{n}\right)$ of Gelfand numbers is the largest injective quasi $s$-number sequence, which satisfies the multiplicativity property $(\mathrm{M})$ :

$$
c_{k+n-1}(S T) \leq c_{k}(S) c_{n}(T), \quad S \in \mathcal{L}(Y, Z), \quad T \in \mathcal{L}_{m}\left(X_{1} \times \cdots \times X_{m}, Y\right) .
$$

The proof of this property is similar to the linear case proof. One should first observe that a multilinear variant of Carl's mixing multiplicativity of an injective $s$-number sequence $\left(s_{n}\right)$ states that, for all $S \in \mathcal{L}(Y, Z)$ and $T \in \mathcal{L}_{m}\left(X_{1} \times \cdots \times X_{m}, Y\right)$,

$$
s_{k+n-1}(S T) \leq c_{k}(T) s_{n}(S), \quad k, n \in \mathbf{N} .
$$

In fact, the mixing multiplicativity property (MI) implies

$$
s_{k+n-1}(S T)=s_{k+n-1}\left(\kappa_{Z} T S\right) \leq a_{k}\left(\kappa_{Z} T\right) s_{n}(S)=c_{k}(T) s_{n}(S), \quad k, n \in \mathbf{N} .
$$

For each $m \in \mathbf{N}$, we define the function $c: \mathcal{L}_{m}\left(X_{1} \times \cdots \times X_{m}\right) \rightarrow[0, \infty)$ by

$$
c(T)=\lim _{n \rightarrow \infty} c_{n}(T) .
$$

The following proposition gives characterization of compactness of multilinear operators in terms of the quantity $c$.

Proposition 5.1. Let $m \geq 2$ and let $X_{1}, \ldots, X_{m}, Y$ be Banach spaces. Then the following statements about an operator $T \in \mathcal{L}_{m}\left(X_{1} \times \cdots \times X_{m}, Y\right)$ are equivalent:

(i) $T$ is compact.

(ii) $c(T)=0$.

(iii) $c\left(T^{\times}\right)=0$.

Proof. Obviously $T$ is compact if, and only if, $\kappa_{Y} T: X_{1} \times \cdots \times X_{m} \rightarrow \ell_{\infty}\left(U_{Y^{*}}\right)$ is compact. Since $\ell_{\infty}\left(U_{Y^{*}}\right)$ has an approximation property, $\kappa_{Y} T$ is an approximable operator and, we may conclude that $T$ is compact if, and only if,

$$
c(T)=\lim _{n \rightarrow \infty} a_{n}\left(\kappa_{Y} T\right)=0 .
$$

This shows that (i) and (iii) are equivalent. To complete the proof, it is enough to recall that $T$ is compact if, and only if, $T^{\times}$is compact.

Our next result shows the relation between Gelfand and Kolmogorov numbers of a multilinear operator $T$ and its adjoint $T^{\times}$.

Theorem 5.1. Let $m \geq 2$ and let $X_{1}, \ldots, X_{m}, Y$ be Banach spaces. Then, for every operator $T \in \mathcal{L}_{m}\left(X_{1} \times \cdots \times X_{m}, Y\right)$ and for each $n \in \mathbf{N}$, we have

(i) $c_{n}\left(T^{\times}\right) \leq d_{n}(T)$,

(ii) $c_{n}(T)=d_{n}\left(T^{\times}\right)$,

(iii) $c_{n}(T) \leq 2 \sqrt{n} c_{n}\left(T^{\times}\right)$.

Proof. (i) For each $1 \leq j \leq m$ let $Q_{j}$ be the canonical metric surjection from $\ell_{1}\left(U_{X_{j}}\right)$ onto $X_{j}$. For abbreviation, let $Q$ stand for the operator

$$
Q_{1} \times \cdots \times Q_{m}: \ell_{1}\left(U_{X_{1}}\right) \times \cdots \times \ell_{1}\left(U_{X_{m}}\right) \rightarrow X_{1} \times \cdots \times X_{m}
$$


defined by for every $\left(\lambda_{1}, \ldots, \lambda_{m}\right) \in \ell_{1}\left(U_{X_{1}}\right) \times \cdots \times \ell_{1}\left(U_{X_{m}}\right)$ by

$$
Q\left(\lambda_{1}, \ldots, \lambda_{m}\right)=\left(Q_{1} \lambda_{1}, \ldots, Q_{m} \lambda_{m}\right) \text {. }
$$

It is easy to verify that $(T Q)^{\times}: Y^{*} \rightarrow \mathcal{L}_{m}\left(\ell_{1}\left(U_{X_{1}}\right) \times \cdots \times \ell_{1}\left(U_{X_{m}}\right)\right)$ factorizes through $\mathcal{L}_{m}\left(X_{1} \times \cdots \times X_{m}\right)$ as follows

$$
(T Q)^{\times}: Y^{*} \stackrel{T^{\times}}{\longrightarrow} \mathcal{L}_{m}\left(X_{1} \times \cdots \times X_{m}\right) \stackrel{\Phi}{\longrightarrow} \mathcal{L}_{m}\left(\ell_{1}\left(U_{X_{1}}\right) \times \cdots \times \ell_{1}\left(U_{X_{m}}\right)\right),
$$

where $\Phi$ is given by

$$
\Phi(S)=S Q, \quad S \in \mathcal{L}_{m}\left(X_{1} \times \cdots \times X_{m}\right) .
$$

Since $Q_{j}$ is a metric surjection for each $1 \leq j \leq m, \Phi$ is a metric injection from $\mathcal{L}_{m}\left(X_{1} \times \cdots \times X_{m}\right)$ into $\mathcal{L}_{m}\left(\ell_{1}\left(U_{X_{1}}\right) \times \cdots \times \ell_{1}\left(U_{X_{m}}\right)\right)$. Combining these with the injectivity of the Gelfand numbers, Proposition 3.2 and Theorem 4.1, one has

$$
c_{n}\left(T^{\times}\right)=c_{n}\left(\Phi T^{\times}\right)=c_{n}\left((T Q)^{\times}\right) \leq a_{n}\left((T Q)^{\times}\right) \leq a_{n}(T Q)=d_{n}(T) .
$$

(ii) Since $\kappa_{Y}: Y \rightarrow \ell_{\infty}\left(U_{Y^{*}}\right)$ is a metric injection, $\left(\kappa_{Y}\right)^{*}: \ell_{\infty}\left(U_{Y^{*}}\right)^{*} \rightarrow Y^{*}$ is a metric surjection. Thus the surjectivity of the Kolgomorov numbers in combination with Proposition 3.2 yields (by $\left(\kappa_{Y} T\right)^{\times}=T^{\times}\left(\kappa_{Y}\right)^{*}$ )

$$
d_{n}\left(T^{\times}\right)=d_{n}\left(T^{\times}\left(\kappa_{Y}\right)^{*}\right) \leq a_{n}\left(T^{\times}\left(\kappa_{Y}\right)^{*}\right)=a_{n}\left(\left(\kappa_{Y} T\right)^{\times}\right) \leq a_{n}\left(\kappa_{Y} T\right)=c_{n}(T) .
$$

To prove the reverse inequality, we use the well-known fact that, for every operator $S \in \mathcal{L}(E, F)$ between Banach spaces $E$ and $F$ (see [11, Proposition 11.7.6]),

$$
c_{n}\left(S^{*}\right) \leq d_{n}(S), \quad n \in \mathbf{N} .
$$

To estimate $c_{n}(T)$ from the above, we apply the equality $\kappa_{Y} T=T^{\times} J$ from Lemma 2.1 and the injectivity of the Gelfand numbers, namely,

$$
c_{n}(T)=c_{n}\left(\kappa_{Y} T\right)=c_{n}\left(T^{\times} J\right) \leq c_{n}\left(\left(T^{\times}\right)^{*}\right) \leq d_{n}\left(T^{\times}\right)
$$

and so this gives the required estimate.

(iii) It is well-known (see, e.g., [4, Proposition 3.8, p. 75]) that for any operator $S: E \rightarrow F$ between Banach spaces and all $n \in \mathbf{N}$,

$$
a_{n}(S) \leq 2 \sqrt{n} c_{n}(S) .
$$

Since $d_{n}\left(T^{\times}\right) \leq a_{n}\left(T^{\times}\right)$, the required estimate follows by (ii).

In the case of any $m$-linear operator $T: X_{1} \times \cdots \times X_{m} \rightarrow Y$ acting between Banach spaces, we also define sequences $\left(\widehat{c}_{n}(T)\right)$ and $\left(\widetilde{c}_{n}(T)\right)$ as follows: $\widehat{c}_{n}(T)$ is to be the infimum of all $\varepsilon>0$, such that there are functionals $B_{i} \in \mathcal{L}_{m}\left(X_{1} \times \cdots \times X_{m}\right)$, $1 \leq i \leq k<n$, which admit an estimate for all $\left(x_{1}, \ldots, x_{m}\right) \in\left(X_{1}, \ldots, X_{m}\right)$,

$$
\left\|T\left(x_{1}, \ldots, x_{m}\right)\right\| \leq \sup _{1 \leq i \leq k}\left|B_{i}\left(x_{1}, \ldots, x_{m}\right)\right|+\varepsilon\left\|x_{1}\right\| \cdots\left\|x_{m}\right\|,
$$

and, respectively, $\widetilde{c}_{n}(T)$ is defined to be the infimum of all $\|S\|$, with $S \in \mathcal{L}_{m}\left(X_{1} \times \cdots\right.$ $\left.\cdot \times X_{m}, Z\right)$ for some Banach space $Z$, such that, there are $B_{i} \in \mathcal{L}_{m}\left(X_{1} \times \cdots \times X_{m}\right)$, $1 \leq i \leq k<n$, which satisfy

$$
\|T x\|_{Y} \leq \sup _{1 \leq i \leq k}\left|B_{i} x\right|+\|S x\|_{Z}, \quad x \in X_{1} \times \cdots \times X_{m} .
$$

To show some properties of the above introduced sequences, we need a characterization of multilinear compact operators in terms of factorization. We will use a characterization of compact linear operators due to Terzioglou [17] (see also [16]; 
in a more precise form, see [8]), which states: If $X, Y$ are Banach spaces, $\varepsilon>0$ is given and $T \in \mathcal{L}(X, Y)$ is compact, then there exists a closed subspace $Z$ of $c_{0}$, such that, $T$ admits a factorization through $Z$ :

$$
T: X \stackrel{B}{\longrightarrow} Z \stackrel{A}{\longrightarrow} Y,
$$

where $B: X \rightarrow Z$ and $A: Z \rightarrow Y$ are compact operators with $\|A\| \leq 1$ and $\|B\| \leq$ $(1+\varepsilon)\|T\|$.

In the multilinear case, we have the following variant.

Theorem 5.2. Let $m \in \mathbf{N}$ and let $X_{1}, \ldots, X_{m}, Y$ be Banach spaces. Given $\varepsilon>0$ and an operator $T \in \mathcal{L}_{m}\left(X_{1} \times \cdots \times X_{m}, Y\right)$, then there exists a closed subspace $Z$ of $c_{0}$, such that, $T$ admits a factorization through $Z$ :

$$
T: X_{1} \times \cdots \times X_{m} \stackrel{B}{\longrightarrow} Z \stackrel{A}{\longrightarrow} Y,
$$

where $B \in \mathcal{L}_{m}\left(X_{1} \times \cdots \times X_{m}, Z\right)$ and $A \in \mathcal{L}(Z, Y)$ are compact operators with $\|A\| \leq 1$ and $\|B\| \leq(1+\varepsilon)\|T\|$.

Proof. It follows from the theory of the projective tensor product analysis that there exist bounded linear operators $\otimes: X_{1} \times \cdots \times X_{m} \rightarrow X_{1} \widehat{\otimes}_{\pi} \cdots \widehat{\otimes}_{\pi} X_{m}$ and $\widetilde{T}: X_{1} \widehat{\otimes}_{\pi} \cdots \widehat{\otimes}_{\pi} X_{m} \rightarrow Y$, such that,

$$
\bigotimes\left(x_{1}, \ldots, x_{m}\right)=x_{1} \otimes \cdots \otimes x_{m}, \quad\left(x_{1}, \ldots, x_{m}\right) \in X_{1} \times \cdots \times X_{m},
$$

and

$$
T=\widetilde{T} \circ \bigotimes \text { with }\|\otimes\| \leq 1,\|\widetilde{T}\| \leq\|T\| .
$$

Using the representation of the projective tensor norm, we deduce that

$$
\widetilde{T}\left(U_{X_{1} \widehat{\otimes}_{\pi} \cdots \widehat{\otimes}_{\pi} X_{m}}\right) \subset \overline{\operatorname{conv}}\left(T\left(U_{X_{1} \times \cdots \times X_{m}}\right)\right) .
$$

Our hypothesis that $T$ is compact in combination with the well-known Mazur theorem yields that $\widetilde{T}$ is a compact operator. To conclude, it is enough to apply the above shown factorization result to $\widetilde{T}$.

Lemma 5.1. Both $\left(\widehat{c}_{n}\right)$ and $\left(\widetilde{c}_{n}\right)$ are injective $s$-number sequences which satisfy the following estimates:

$$
\widehat{c}_{n}(T) \leq \widetilde{c}_{n}(T) \leq c_{n}(T), \quad n \in \mathbf{N} .
$$

Proof. It is easy to check that the properties (S1)-(S3) are satisfied for both sequences $\left(\widehat{c}_{n}\right)$ and $\left(\widetilde{c}_{n}\right)$. We claim that the rank property (S3) holds for $\left(\widetilde{c}_{n}\right)$. To see this, fix $T \in \mathcal{L}_{m}\left(X_{1} \times \cdots \times X_{m}, Y\right)$ with $\operatorname{rank}(T)<n$. Since

$$
T: X_{1} \times \cdots \times X_{m} \rightarrow[\operatorname{Im}(T)]
$$

is compact, it follows by Theorem 5.2 that there exists a closed subspace $Z$ of $c_{0}$ such that $T$ admits a factorization through $Z$,

$$
T: X_{1} \times \cdots \times X_{m} \stackrel{B}{\longrightarrow} Z \stackrel{A}{\longrightarrow}[\operatorname{Im}(T)]
$$

with $\|A\| \leq 1$ and $\|B\| \leq(1+\varepsilon)\|T\|$.

Since $\operatorname{rank}(T)=\operatorname{dim}([\operatorname{Im}(T)])<n, \operatorname{rank}(A)<n$ and so $c_{n}(A)=0$. Thus for every $\varepsilon>0$ there are functionals $z_{i}^{*} \in Z^{*}, 1 \leq k<n$, which admit an estimate

$$
\|A z\|_{Y} \leq \sup _{1 \leq i \leq k}\left|\left\langle z, z_{i}^{*}\right\rangle\right|+\varepsilon\|z\|_{Z}, \quad z \in Z .
$$


This implies that for $B_{i}:=z_{i}^{*} \circ B \in \mathcal{L}_{m}\left(X_{1} \times \cdots \times X_{m}\right), 1 \leq i \leq k<n$, and $S:=\varepsilon B \in \mathcal{L}_{m}\left(X_{1} \times \cdots \times X_{m}, Z\right)$ we have

$$
\|T x\|_{Y}=\|A(B x)\|_{Y} \leq \sup _{1 \leq i \leq k}\left|B_{i} x\right|+\|S x\|_{Z}, \quad x \in X_{1} \times \cdots \times X_{m} .
$$

Consequently we obtain

$$
\widetilde{c}_{n}(T) \leq\|S\|=\varepsilon\|B\| \leq \varepsilon(1+\varepsilon)\|T\| .
$$

Since $\varepsilon>0$ is arbitrary, $\widetilde{c}_{n}(T)=0$ and so the claim is proved.

Obviously both $\left(\widehat{c}_{n}\right)$ and $\left(\widetilde{c}_{n}\right)$ are injective $s$-number sequences, which satisfy $\widehat{c}_{n}(T) \leq \widetilde{c}_{n}(T)$ for all $T \in \mathcal{L}_{m}\left(X_{1} \times \cdots \times X_{m}, Y\right)$ and $n \in \mathbf{N}$. Since the sequence of Gelfand numbers is the largest injective $s$-number sequence, the proof is complete.

Pietsch in [11, p. 149] proved that the $n$-th Gelfand number $c_{n}(T)$ of an operator between Banach spaces $X$ and $Y$ allows the representation

$$
c_{n}(T)=\inf \left\{\left\|T I_{F}\right\| ; F \subset X, \operatorname{codim}(F)<n\right\},
$$

where $I_{F}$ is the inclusion map from $F$ into $X$.

Fix a positive integer $m \geq 2$. Let $X_{1}, \ldots, X_{m}$ and $Y$ be arbitrary Banach spaces. Motivated by Pietsch's result, for any bounded $m$-linear operator $T: X_{1} \times \cdots \times X_{m} \rightarrow$ $Y$ and $\left(n_{1}, \ldots, n_{m}\right) \in \mathbf{N}^{m}$, we define a sequence $\left(\bar{c}_{\left(n_{1}, \ldots, n_{m}\right)}(T)\right)$ by

$$
\bar{c}_{\left(n_{1}, \ldots, n_{m}\right)}(T)=\inf \left\{\left\|T I_{F_{1} \times \cdots \times F_{m}}\right\| ; F_{i} \subset X_{i}, 1 \leq i \leq m, \operatorname{codim}\left(F_{i}\right)<n_{i}\right\},
$$

where $F_{i}$ is closed subspaces of $X_{i}$ for each $1 \leq j \leq m$.

Unfortunately in the multilinear case, the relations between the sequence $\left(c_{n}(T)\right)$ of Gelfand numbers and the sequence $\left(\bar{c}_{\left(n_{1}, \ldots, n_{m}\right)}(T)\right)$ seems to be generally complicated.

We show some relationships between mentioned sequences for $m$-linear operators defined on the product of finite dimensional spaces. We need some definitions and preliminary results.

Suppose $m \geq 2$ and $F_{1}, \ldots, F_{m}$ are closed subspaces, respectively, of the Banach spaces $X_{1}, \ldots, X_{m}$. Throughout the rest of the paper we put

$$
\left(F_{1} \times \cdots \times F_{m}\right)^{\circ}:=\left\{T \in \mathcal{L}_{m}\left(X_{1} \times \cdots \times X_{m}\right) ; T x=0 \text { for all } x \in F_{1} \times \cdots \times F_{m}\right\} .
$$

Obviously $\left(F_{1} \times \cdots \times F_{m}\right)^{\circ}$ is a closed subspace of $\mathcal{L}_{m}\left(X_{1} \times \cdots \times X_{m}\right)$. For every operator $T \in \mathcal{L}_{m}\left(X_{1} \times \cdots \times X_{m}\right), \bar{T}:=T+\left(F_{1} \times \cdots \times F_{m}\right)^{\circ}$ denotes an element of the quotient space $\mathcal{L}_{m}\left(X_{1} \times \cdots \times X_{m}\right) /\left(F_{1} \times \cdots \times F_{m}\right)^{\circ}$. The quotient map from $\mathcal{L}_{m}\left(X_{1} \times \cdots \times X_{m}\right)$ onto $\mathcal{L}_{m}\left(X_{1} \times \cdots \times X_{m}\right) /\left(F_{1} \times \cdots \times F_{m}\right)^{\circ}$ will be denoted by $Q_{\left(F_{1} \times \cdots \times F_{m}\right)^{\circ}}$.

Lemma 5.2. Let $m \geq 2$ and let $F_{1}, \ldots, F_{m}$ be closed subspaces respectively of the Banach spaces $X_{1}, \ldots, X_{m}$, such that for every $T \in \mathcal{L}_{m}\left(F_{1} \times \cdots \times F_{m}\right)$ there exists $\widetilde{T} \in \mathcal{L}_{m}\left(X_{1} \times \cdots \times X_{m}\right)$ with $\left.\widetilde{T}\right|_{F_{1} \times \cdots \times F_{m}}=T$ and $\|\widetilde{T}\| \leq M\|T\|$ for some numerical constant $M \geq 1$. Then the operator $\Psi: \mathcal{L}_{m}\left(X_{1} \times \cdots \times X_{m}\right) /\left(F_{1} \times \cdots \times F_{m}\right)^{\circ} \rightarrow$ $\mathcal{L}_{m}\left(F_{1} \times \cdots \times F_{m}\right)$ given by

$$
\Psi(\bar{T}):=\left.T\right|_{F_{1} \times \cdots \times F_{m}}, \quad \bar{T} \in \mathcal{L}_{m}\left(X_{1} \times \cdots \times X_{m}\right) /\left(F_{1} \times \cdots \times F_{m}\right)^{\circ}
$$

is a linear isomorphism such that $M^{-1} \leq\|\Psi\| \leq 1$. 
Proof. It is clear that $\Psi$ is linear and one-to-one. Let $T \in \mathcal{L}_{m}\left(X_{1} \times \cdots \times X_{m}\right)$. Then for all $S \in\left(F_{1} \times \cdots \times F_{m}\right)^{\circ}$, we have

$$
\|\Psi(\bar{T})\|=\left\|\left.T\right|_{F_{1} \times \cdots \times F_{m}}\right\|=\left\|\left.(T+S)\right|_{F_{1} \times \cdots \times F_{m}}\right\| \leq\|T+S\| .
$$

In consequence $\|\Psi\| \leq 1$.

To complete the proof, for a given $B \in \mathcal{L}_{m}\left(F_{1} \times \cdots \times F_{m}\right)$, our hypothesis implies that there exists $T \in \mathcal{L}_{m}\left(X_{1} \times \cdots \times X_{m}\right)$ with $\|T\| \leq M\|B\|$, such that, $T$ is $B$ on $F_{1} \times \cdots \times F_{m}$. In particular this implies that

$$
\Psi(\bar{T})=\left.T\right|_{F_{1} \times \cdots \times F_{m}}=B
$$

and so $\Psi$ is onto. We also have

$$
M^{-1}\|\bar{T}\| \leq M^{-1}\|T\| \leq\|B\|=\|\Psi(\bar{T})\|,
$$

which gives $M^{-1} \leq\|\Psi\|$.

Theorem 5.3. Let $m \geq 2, X_{1}, \ldots, X_{m}, Y$ be Banach spaces, and let $F_{1}, \ldots, F_{m}$ be closed subspaces of the Banach spaces $X_{1}, \ldots, X_{m}$, respectively, which satisfy hypotheses of Lemma 5.2. Then for any operator $T \in \mathcal{L}_{m}\left(X_{1} \times \cdots \times X_{m}, Y\right)$,

$$
\left\|Q_{\left(F_{1} \times \cdots \times F_{m}\right)} T^{\times}\right\| \leq M\left\|T I_{F_{1} \times \cdots \times F_{m}}\right\|,
$$

where $I_{F_{1} \times \cdots \times F_{m}}$ is the inclusion map from $F_{1} \times \cdots \times F_{m}$ into $X_{1} \times \cdots \times X_{m}$.

Proof. Let $R: \mathcal{L}_{m}\left(X_{1} \times \cdots \times X_{m}\right) \rightarrow \mathcal{L}_{m}\left(F_{1} \times \cdots \times F_{m}\right)$ be the restriction map defined by

$$
R(S):=\left.S\right|_{F_{1} \times \cdots \times F_{m}}, \quad S \in \mathcal{L}_{m}\left(X_{1} \times \cdots \times X_{m}\right) .
$$

For any $T \in \mathcal{L}_{m}\left(X_{1} \times \cdots \times X_{m}\right)$, we have

$$
R T^{\times}: Y^{*} \rightarrow \mathcal{L}_{m}\left(F_{1} \times \cdots \times F_{m}\right) .
$$

Since for all $y^{*} \in Y^{*}$,

$$
R T^{\times}\left(y^{*}\right)=R\left(T^{\times} y^{*}\right)=R\left(y^{*} \circ T\right)=\left.\left(y^{*} \circ T\right)\right|_{F_{1} \times \cdots \times F_{m}}=y^{*} \circ\left(T I_{F_{1} \times \cdots \times F_{m}}\right),
$$

it follows that

$$
\left\|R T^{\times}\right\|=\left\|T I_{F_{1} \times \cdots \times F_{m}}\right\| .
$$

To conclude observe that $R=\Psi Q_{\left(F_{1} \times \cdots \times F_{m}\right)^{\circ}}$ where

$$
\Psi: \mathcal{L}_{m}\left(X_{1} \times \cdots \times X_{m}\right) /\left(F_{1} \times \cdots \times F_{m}\right)^{\circ} \rightarrow \mathcal{L}_{m}\left(F_{1} \times \cdots \times F_{m}\right)
$$

is an operator defined as in Lemma 5.2 and apply 5.2.

Theorem 5.4. Let $m \geq 2$ and let $X_{1}, \ldots, X_{m}, Y$ be Banach spaces such that $N_{i}=\operatorname{dim}\left(X_{i}\right)<\infty$ for each $1 \leq i \leq m$. Assume $X_{1} \times \cdots \times X_{m}$ has the multilinear extension property with $M$. Then for all $T \in \mathcal{L}_{m}\left(X_{1} \times \cdots \times X_{m}, Y\right)$,

$$
c_{n}(T) \leq M \bar{c}_{\left(k_{1}, \ldots, k_{m}\right)}(T), \quad 1 \leq k_{i}<N_{i}, \quad 1 \leq i \leq m,
$$

where $n \geq N_{1} \cdots N_{m}-\left(N_{1}-k_{1}\right) \cdots\left(N_{m}-k_{m}\right)$.

Proof. Fix $\varepsilon>0$. Then for each $1 \leq i \leq m$ there exists a closed subspace $F_{i}$ of $X_{i}$, such that $\operatorname{codim}\left(F_{i}\right)<k_{i}$ and

$$
\left\|T I_{F_{1} \times \cdots \times F_{m}}\right\| \leq \bar{c}_{\left(k_{1}, \ldots, k_{m}\right)}(T)+\varepsilon / M .
$$


It follows from Lemma 5.2 that $\operatorname{codim}\left(\left(F_{1} \times \cdots \times F_{m}\right)^{\circ}\right)=\operatorname{dim}\left(\mathcal{L}_{m}\left(F_{1} \times \cdots \times F_{m}\right)\right)<\infty$. Since $\operatorname{dim}\left(F_{i}\right)>N_{i}-k_{i}$ for each $1 \leq i \leq m$,

$$
\begin{aligned}
N:=\operatorname{dim}\left(\left(F_{1} \times \cdots \times F_{m}\right)^{\circ}\right) & =\operatorname{dim}\left(\mathcal{L}_{m}\left(X_{1} \times \cdots \times X_{m}\right)\right)-\operatorname{dim}\left(\mathcal{L}_{m}\left(F_{1} \times \cdots \times F_{m}\right)\right) \\
& =\operatorname{dim}\left(X_{1}\right) \cdots \operatorname{dim}\left(X_{m}\right)-\operatorname{dim}\left(F_{1}\right) \cdots \operatorname{dim}\left(F_{m}\right) \\
& <N_{1} \cdots N_{m}-\left(N_{1}-k_{1}\right) \cdots\left(N_{m}-k_{m}\right) .
\end{aligned}
$$

Combining Theorems 5.1 and 5.3 and with $(*)$ yields

$$
\begin{aligned}
c_{n}(T) & =d_{n}\left(T^{\times}\right) \leq d_{N}\left(T^{\times}\right) \leq\left\|Q_{\left(F_{1} \times \cdots \times F_{m}\right)^{\circ}} T^{\times}\right\| \\
& \leq M\left\|T I_{F_{1} \times \cdots \times F_{m}}\right\| \leq M \bar{c}_{\left(k_{1}, \ldots, k_{m}\right)}(T)+\varepsilon .
\end{aligned}
$$

Since $\varepsilon>0$ is arbitrary the proof, the required estimate follows.

It is clear that if $H_{1}, \ldots, H_{m}$ are Hilbert spaces, then $H_{1} \times \cdots \times H_{m}$ has the multilinear extension property. Let us remark that Hayden's extension theorem [7, Theorem 7] gives more precise result that $M\left(H_{1} \times \cdots \times H_{m}\right)=1$. In consequence, we obtain the following.

Corollary 5.1. Let $m \geq 2$ and $H_{1}, \ldots, H_{m}$ be Hilbert spaces and let $Y$ be a Banach space such that $N_{i}=\operatorname{dim}\left(H_{i}\right)<\infty$ for each $1 \leq i \leq m$. Then for all $T \in \mathcal{L}_{m}\left(H_{1} \times \cdots \times H_{m}, Y\right)$,

$$
c_{n}(T) \leq \bar{c}_{\left(k_{1}, \ldots, k_{m}\right)}(T), \quad 1 \leq k_{i}<N_{i}, \quad 1 \leq i \leq m,
$$

where $n \geq N_{1} \cdots N_{m}-\left(N_{1}-k_{1}\right) \cdots\left(N_{m}-k_{m}\right)$.

To show applications to bilinear operators we recall that a Banach $X$ has type $p, 1<p \leq 2$, provided there exists a constant $C>0$, such that, for every choice of finitely many elements $x_{1}, \ldots, x_{n} \in X$

$$
\left(\int_{0}^{1}\left\|\sum_{k=1}^{n} r_{k}(t) x_{k}\right\|^{2} d t\right)^{1 / 2} \leq C\left(\sum_{k=1}^{n}\left\|x_{k}\right\|^{p}\right)^{1 / p},
$$

where $\left(r_{n}\right)$ is the sequence of the Rademacher functions. The least constant $C$ in the above inequality is called the type constant of $X$ and is denoted by $T_{p}(X)$.

Maurey's celebrated extension theorem (see [3, pp. 246-248]) implies that $X_{1} \times X_{2}$ has the bilinear extension property with $M$ for arbitrary Banach spaces $X_{1}$ and $X_{2}$ of type 2 , where $M$ depends type constants $T_{2}\left(X_{1}\right)$ and $T_{2}\left(X_{2}\right)$ of $X_{1}$ and $X_{2}$, respectively. Examples of Banach spaces with type 2 are $L_{p}$-spaces for $2 \leq p<\infty$.

We conclude with the following corollary.

Corollary 5.2. Let $X_{1}, X_{2}$ and $Y$ be Banach spaces. If $X_{1}$ and $X_{2}$ are finite dimensional spaces of type 2 with $\operatorname{dim}\left(X_{1}\right)=N_{1}$ and $\operatorname{dim}\left(X_{2}\right)=N_{2}$, then there exists a constant $C>0$, such that, for every bounded bilinear operator $T: X_{1} \times X_{2} \rightarrow Y$,

$$
c_{n}(T) \leq C \bar{c}_{\left(k_{1}, k_{2}\right)}(T), \quad 1 \leq k_{i}<N_{i}, \quad 1 \leq i \leq 2,
$$

where $n \geq N_{1} N_{2}-\left(N_{1}-k_{1}\right)\left(N_{2}-k_{2}\right)$.

\section{References}

[1] CARL, B.: On $s$-numbers, quasi $s$-numbers, $s$-moduli and Weyl inequalities of operators in Banach spaces. - Rev. Mat. Complut. 23:2, 2010, 467-487.

[2] Carl, B., and I. Stephani: Entropy, compactness and the approximation of operators. Cambridge Univ. Press, Cambridge, 1990. 
[3] Diestel, J., H. Jarchow, and A. Tonge: Absolutely summing operators. - Cambridge Univ. Press, 1995.

[4] Edmunds, D. E., and W. D. Evans: Spectral theory and differential operators. - Oxford Math. Monogr., Oxford Science Publications, The Clarendon Press, Oxford Univ. Press, New York, 1987.

[5] Edmunds, D. E., and H.-O. Tylli: On the entropy numbers of an operator and its adjoint. - Math. Nachr. 126, 1986, 231-239.

[6] Fernández-Unzueta, M.: Extension of multilinear maps defined on subspaces. - Israel J. Math. 188, 2012, 301-322.

[7] Hayden, T. L.: The extension of bilinear functionals. - Pacific J. Math. 22:1, 1967, 99-108.

[8] John, K.: Some remarks on compact maps in Banach spaces. - Czechoslovak Math. J. 23:2, 1973, 177-182.

[9] König, H.: Eigenvalue distribution of compact operators. - Oper. Theory Adv. Appl. 16, Birkhäuser, Basel, 1986.

[10] Pietsch, A.: s-numbers of operators in Banach spaces. - Studia Math. 51, 1974, 201-223.

[11] Pietsch, A.: Operator ideals. - North-Holland, Amsterdam, 1980.

[12] Pietsch, A.: Ideals of multilinear functionals (designs of a theory). - In: Proceedings of the Second International Conference on Operator Algebras, Ideals, and Their Applications in Theoretical Physics (Leipzig, 1983), Teubner-Texte Math. 67, Teubner, Leipzig, 1984, 185-199.

[13] Pietsch, A.: Eigenvalues and s-numbers. - Cambridge Stud. Adv. Math. 13, Cambridge Univ. Press, Cambridge, 1987.

[14] PISIER, G.: Factorization of operators and geometry of Banach spaces. - CBMS Regional Conference Series in Mathematics 60, Amer. Math. Soc., Providence, R.I., 1986.

[15] Ramanujan, M. S., and E. Schock: Operator ideals and space of bilinear operators. - Linear Multilinear Algebra 18, 1985, 307-318.

[16] Randtke, D. J.: A factorization theorem for compact operators. - Proc. Amer. Math. Soc. 34, 1972, 201-202.

[17] Terzioglou, T.: A characterization of compact linear mappings. - Arch. Math. 22, 1972, $76-78$.

Received 10 December 2012 • Accepted 11 February 2013 